\title{
Syndrome de Gardner avec traitement chirurgical des ostéomes multiples. A propos d'un cas
}

\section{Surgical management of osteomas associated with Gardner's syndrome: a case report}

\author{
NADIA BEN LAGHA ${ }^{1,2}$, JEAN-MARC GALÉAZZI' ${ }^{1}$ PASCAL OXEDA ${ }^{3}$, YORAM BOUHNIK ${ }^{4}$, LOUIS MAMAN²
}

\section{RÉSUMÉ}

Le syndrome de Gardner est caractérisé principalement par une polypose gastrointestinale héréditaire, où la dégénérescence maligne constante des polypes rectocoliques impose une détection précoce et un suivi régulier. II associe, outre une polypose rectocolique disséminée, des kystes sébacés multiples, des lipomes souscutanés, des tumeurs fibreuses ou musculaires rétropéritonéales ou intramésentériques, et des ostéomes bénins le plus souvent de localisation crânienne et maxillaire. L'odontologiste peut jouer un rôle important dans le diagnostic de cette affection par le dépistage des lésions osseuses ou muqueuses. Le cas de syndrome de Gardner rapporté comporte de nombreux ostéomes maxillaires qui ont nécessité un traitement chirurgical. (Med Buccale Chir Buccale 2003; 9:177-84)

mots clés: Ostéome, Syndrome de Gardner

médecine buccale chirurgie buccale

VOL. $9, N^{\circ} 3$ 2003

page 177

\section{SUMMARY}

Gardner's syndrome is an autosomal dominant condition consisting of multiple soft and hard tissue tumors and multiple intestinal polyps with a marked tendency to rapid malignant degeneration. Since the Gardner'syndrome has prominent oral manifestations and since these manifestations characteristically precede colonic polyposis by several years, dentists may have an important role in the early diagnosis of this condition. A case of Gardner's syndrome with multiple osteomas of the jaw is reported. Since function as well as aesthetics were compromised surgical excision, wich was performed, is reported. (Med Buccale Chir Buccale 2003; 9: 177-84)

key words: Osteoma, Gardner syndrome

\footnotetext{
Service d'Odontologie, Hôpital Max Fourestier, 403, av. de la République, 92000 Nanterre

2 Faculté de Chirurgie Dentaire, 1 rue Maurice Arnoux, Montrouge.

${ }^{3}$ Service de Chirurgie Maxillo-Faciale, Hôpital de Saint-Cloud, St-Cloud

${ }^{4}$ Service de Gastro-Entérologie, Hôpital Lariboisière, Paris
}

Demande de tirés à part :

N. Ben Lagha Hôpital Max Fourestier 403, av. de la République 92000 Nanterre 
médecine

buccale

chirurgie

buccale

VOL. $9, \mathrm{~N}^{\circ} 3$ 2003

page 178
Le syndrome de Gardner, ou polypose adénomateuse familiale, a été décrit en 1912 par Devic et Bussy [1] mais c'est entre 1950 et 1954 que Gardner en précise la transmission génétique [2]. Ce syndrome résulte d'une anomalie de développement des trois feuillets embryonnaires primitifs: l'ectoderme, le mésoderme et l'endoderme. II associe une polypose rectocolique disséminée qui évolue constamment vers la dégénérescence maligne, des kystes sébacés multiples, des lipomes sous-cutanés, des tumeurs fibreuses ou musculaires rétropéritonéales ou intramésentériques, et des ostéomes bénins ayant le plus souvent une localisation crânienne et maxillaire.

Ce syndrome représente une affection héréditaire autosomique dominante rare, liée à une mutation du gène Adenomatous polyposis coli (APC), situé sur le bras long du chromosome 5 (5q21-q22) [3]. Sa fréquence est estimée entre 1 pour 6000 et 1 pour 13000 naissances. Ces mutations génétiques sont généralement transmises par l'un des parents mais elles surviennent de novo dans un tiers des cas. La quasi-totalité des mutations du gène $A P C$ a pour conséquence la production d'une protéine tronquée [3,4,5]. Le type de mutation détermine la gravité du phénotype. Ainsi, dans ce syndrome où plus de 200 mutations du gène $A P C$ ont été rapportées, on peut trouver des manifestations cliniques différentes avec des degrés d'atteinte variables. Les manifestations cliniques sont multiples et peuvent atteindre le cerveau, l'estomac, les intestins, l'épiderme, le système endocrinien, les yeux, le squelette et la cavité buccale. La prolifération de l'endoblaste est responsable de la polypose adénomateuse rectocolique, les anomalies de l'ectoblaste se traduisent par le développement de kystes épidermoïdes et sébacés et les anomalies du mésoderme par des ostéomes, des lipomes et des fibromes. Les anomalies de l'endoderme prédominent dans le syndrome de Gardner, et le diagnostic est posé en présence de multiples polypes adénomateux touchant le colon et le rectum. Ils apparaissent fréquemment vers la puberté, plus rarement avant (entre 13 et 31 ans), et conduisent à un cancer colorectal dans presque $100 \%$ des cas avant 40 ans. L'âge moyen d'apparition des tumeurs malignes se situent vers 37 ans mais on estime qu'à 30 ans $50 \%$ des patients présentent déjà une transformation maligne [3]. Chez les enfants issus d'un parent atteint, il est proposé une coloscopie complète annuelle dès la puberté jusqu'à la coloprotectomie. Des polypes extracoliques sont observés dans le tractus gastro-intestinal supérieur à l'exception de l'œsophage. L'incidence des cancers du duodénum, notamment dans la région de l'ampoule de Vater, est augmentée. Les patients peuvent aussi présenter des tumeurs desmoïdes, ou fibromatoses agressives. Ces tumeurs sont généralement considérées comme des tumeurs fibreuses bénignes. Elles peuvent apparaître dans différentes régions de l'organisme mais elles se développent principalement dans la région abdominale. Les tumeurs desmoïdes surviennent le plus souvent sur la cicatrice abdominale lorsqu'il y a eu une résection intestinale. Malgré leur bénignité, elles ont un taux de récidives élevées (environ $65 \%$ ) [6]. Sur le revêtement cutané, environ la moitié des patients présentent des kystes épidermoïdes ou sébacés, localisés indifféremment sur le tronc, les membres, le visage ou la tête. Ces kystes apparaissent vers l'âge de 13 ans, ce qui peut permettre de dépister ce syndrome [3]. On peut trouver également des tumeurs desmoïdes, des lipomes et des fibromes intéressant le revêtement cutané. Ces malades peuvent développer des néoplasies endocriniennes, la plus fréquente étant le carcinome papillaire ou folliculaire de la thyroïde; ces néoplasies sont non fonctionnelles ou occultes mais souvent multifocales [7,8]. L'atteinte oculaire se traduit par une hypertrophie congénitale de l'épithélium pigmentaire de la rétine. Elle constitue un marqueur clinique du syndrome de Gardner lorsqu'aucune autre manifestation n'est encore apparue [9]. Le plus souvent, l'atteinte est unilatérale et isolée mais on peut observer des lésions multiples et bilatérales. L'examen du fond de l'œil montre une hypertrophie de l'épithélium pigmentaire dans près de $90 \%$ des syndromes de Gardner [10].

L'importance du dépistage précoce de ce syndrome apparaît évidente si l'on veut prévenir ses principaux aspects évolutifs. 


\section{OBSERVATION}

Un patient, âgé de 20 ans, est adressé en avril 2001 par le Service de gastro-entérologie, pour le traitement de nombreux ostéomes des maxillaires. Ce patient est hospitalisé en octobre 1998 afin de pratiquer une coloscopie pour suspicion de polypose adénomateuse familiale ; le père présente un syndrome de Gardner avec des manifestations extraintestinales associées comme des ostéomes des membres, du crâne et des maxillaires. Une analyse génétique, réalisée en 1997, met en évidence sur l'exon 15 , codon 15-17, la mutation du gène APC chez les deux enfants de la famille, et donc chez ce jeune patient. La première coloscopie, réalisée en octobre 1998, s'est révélée normale. Un traitement par Arthrocine ${ }^{\circledR}$ est prescrit pour ralentir la cinétique d'apparition des polypes adénomateux, mais le traitement est mal suivi. Une $2^{\mathrm{e}}$ coloscopie, pratiquée en novembre 1999, retrouve plus de dix polypes adénomateux comportant un contingent tubulovilleux avec dysplasie de bas grade. Une coloprotectomie suivie d'anastomose iléoanale a été programmée pour être réalisée dans un délai de 2 ans.

Le motif de la consultation est d'ordre esthétique: de multiples ostéomes mandibulaires présents depuis l'âge de 12 ans ont, depuis 1999, augmenté de taille, entraînant un handicap morphologique majeur, ayant perturbé l'adolescence de ce patient. A l'examen exobuccal, on note la présence de multiples formations nodulaires situées dans la région génienne, droite et gauche, et dans la région sousmandibulaire (Fig. 1). La palpation révèle que ces tumeurs sont dures, bien limitées, non adhérentes au plan cutané et font corps avec la corticale mandibulaire. Les aires ganglionnaires associées sont libres. A l'examen endobuccal, aucune lésion n'est décelée. L'examen tomodensitométrique montre de multiples ostéomes mandibulaires, de différentes tailles, situés sur les faces externe et interne de la mandibule. Les ostéomes mandibulaires, les plus volumineux, présentent un aspect polylobé et atteignent 20 mm de diamètre (Fig. 2). Le traitement proposé au patient est l'exérèse des ostéomes les

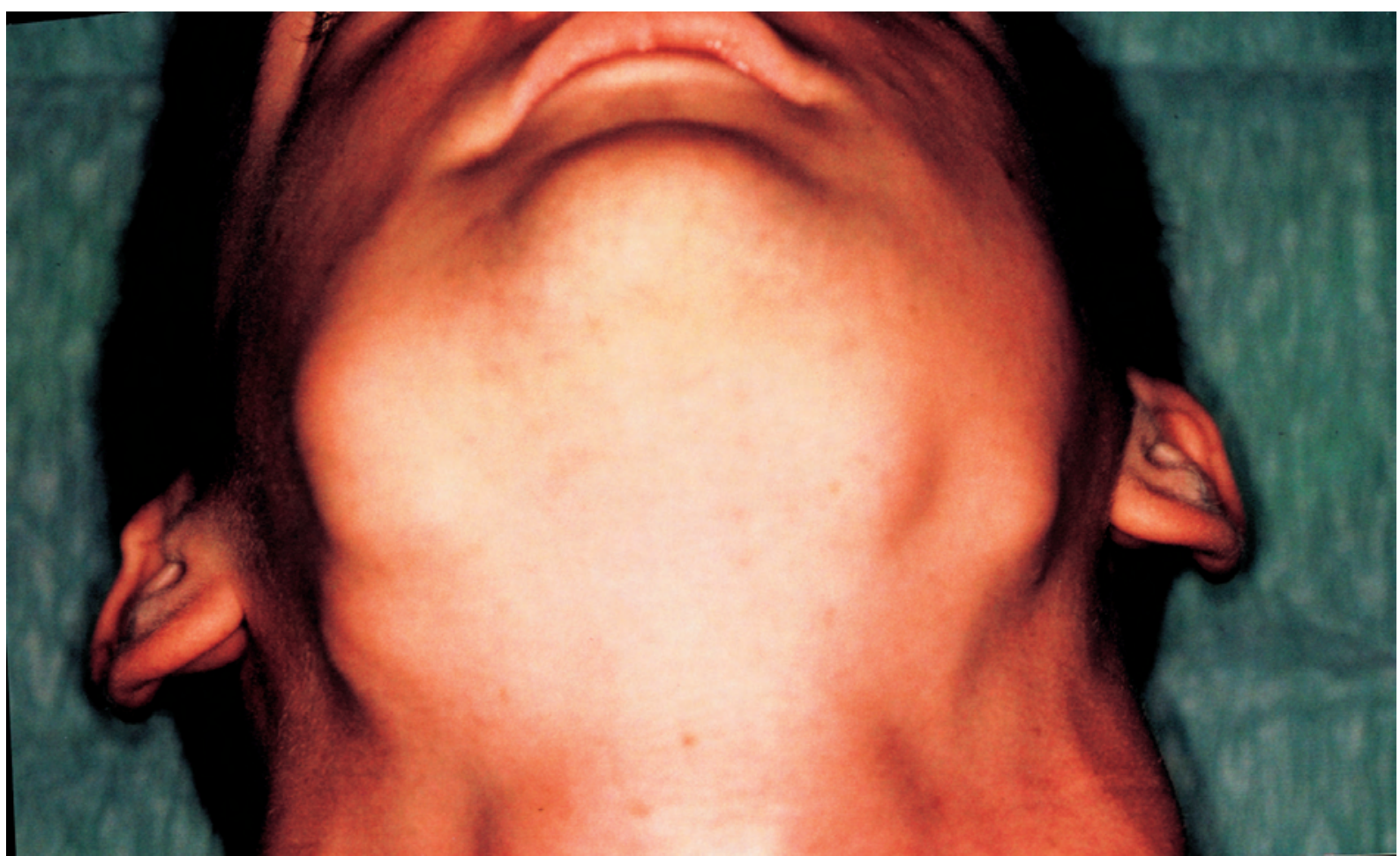

médecine

buccale

chirurgie

buccale

VOL. $9, \mathrm{~N}^{\circ} 3$ 2003

page 179

Figure 1 : Multiples formations nodulaires intéressant les régions jugales droite et gauche, et la région sous-mandibulaire.

Multiple soft tissue lesions in sous-mandibular and in left and right jugal area. 


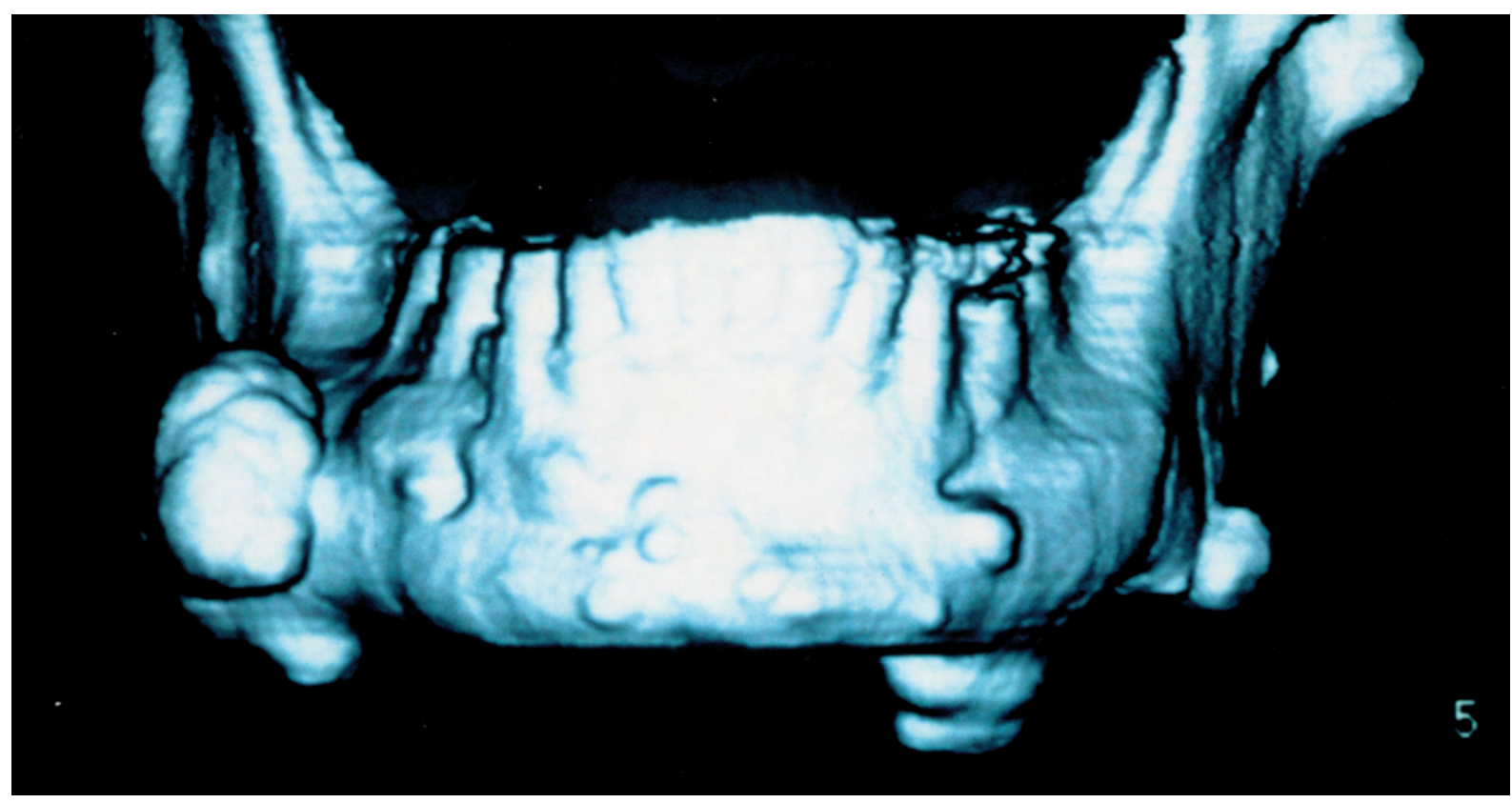

médecine

buccale

chirurgie

buccale

VOL. $9, \mathrm{~N}^{\circ} 3$ 2003

page 180
Figure 2 : Examen tomodensitométrique montrant de multiples ostéomes mandibulaires de différentes tailles, siégeant sur la corticale externe et le bord basilaire de la mandibule. Les ostéomes mandibulaires les plus volumineux présentent un aspect polylobé et atteignent $20 \mathrm{~mm}$ de diamètre.

A preoperative Ct-scan showing a lot of mandibular osteomas of various size localized to the mandibule. The biggest osteomas are polylobulous.

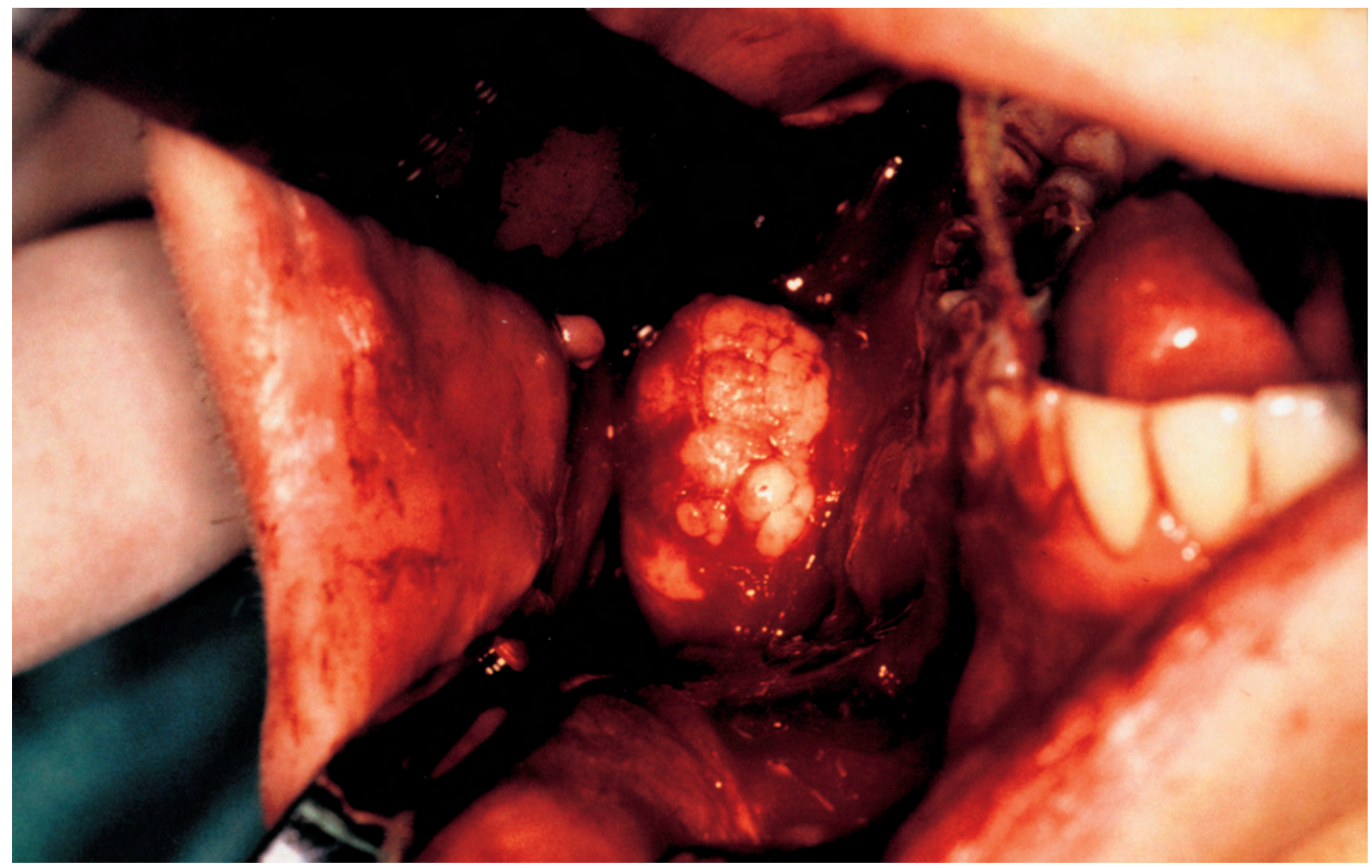

Figure 3 : Voie d'abord endo-buccale avec un lambeau mucopériosté qui permet l'exposition du corps de la mandibule de la région canine à la région molaire, en respectant l'émergence du nerf mentonnier. Surgical excisin of the osteomas using a mucoperiostal flap. 
plus volumineux par voie endo- et exo-buccale sous anesthésie générale.

Par voie endobuccale, un lambeau mucopériosté permet l'exposition du corps de la mandibule depuis la région canine jusqu'à la région molaire, en respectant l'émergence du nerf mentonnier. Ce lambeau découvre les ostéomes localisés sur la face externe du corps mandibulaire en regard des régions prémolaire et molaire (Fig. 3). Après résection de ces ostéomes avec une microscie, la régularisation est réalisée à la fraise. Les ostéomes situés sur le bord basilaire de la mandibule, à gauche et à droite, sont réséqués par un abord cutané sous-mandibulaire. Les ostéomes de petite taille, mis en évidence par l'examen tomodensitométrique, sont laissés en place. L'ostéome le plus postérieur situé sur la face interne de l'angle mandibulaire n'a pas été réséqué car son abord chirurgical est difficile; de plus, il n'occasionne aucune gène fonctionnelle ou esthétique pour le patient. La suture des voies d'abord cutanées cervicales gauche et droite est pratiquée en deux plans tandis que la suture de la voie d'abord endo-buccale est faite en un plan. Les pièces d'exérèse sont adressées au laboratoire d'anatomopathologie. Elles forment un nodule osseux dense où l'os haversien est constitué de canaux de taille inégale, sans liseré ostéoblastique ou ostéoclastique (Fig. 4). Le centre du nodule est occupé par une moelle fibroblastique bien vascularisée, organisée en travées. Le patient est revu une semaine plus tard pour déposer les sutures (Fig. 5). Les suites opé- ratoires sont simples. Le résultat esthétique, à court terme et à un an, est jugé satisfaisant par le patient et sa famille (Fig. 6). L'examen tomodensitométrique réalisé un an après l'intervention ne montre aucune récidive et l'ostéome non réséqué n'a pas augmenté de volume (Fig. 7).

\section{COMMENTAIRES}

Le syndrome de Gardner, dans sa description succincte, est caractérisé par des polypes colorectaux, par des ostéomes (énostoses et exostoses) qui peuvent concerner tous les os mais ils intéressent surtout le calvaria. Sur les os longs, les ostéomes sont petits, d'évolution lente, et souvent limités à un épaississement cortical sous-périosté comme on peut en observer dans certaines ostéites. Sur les os de la face, les ostéomes peuvent apparaître n'importe où mais ils touchent principalement l'os frontal, le maxillaire et l'angle de la mandibule [11,12,13,14]. Dans le cas rapporté, les ostéomes intéressent uniquement la mandibule alors que, chez son père, ils sont situés principalement sur l'os frontal. Le phénotype peut être différent pour les sujets appartenant à une même famille. Généralement, les ostéomes sont de taille modérée, avec un potentiel de croissance réduit. Leur nombre et leur taille peuvent augmenter avec l'âge; des contrôles de l'évolution à long terme sont préconisés [15]. Le nombre moyen d'ostéomes volumineux chez les patients présentant un syndrome
Figure 4 : Coupe histologique montrant un nodule osseux dense constitué par un os haversien dont les canaux apparaissent de taille inégale, sans liseré ostéoblastique ou ostéoclastique (H\&E, x 25). Histological section of biopsy specimen revealing a haversien bone without osteoblast or osteoclast cell (H\&E, x25).

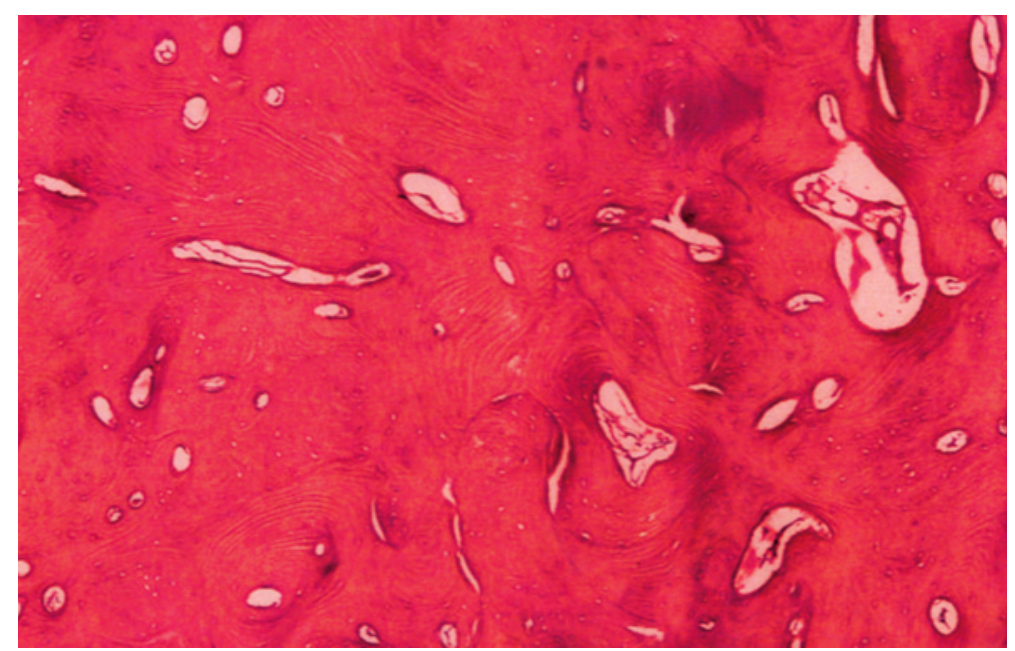

médecine

buccale

chirurgie

buccale

VOL. $9, N^{\circ} 3$ 2003

page 181 
médecine

buccale

chirurgie

buccale

VOL. $9, \mathrm{~N}^{\circ} 3$ 2003

page 182

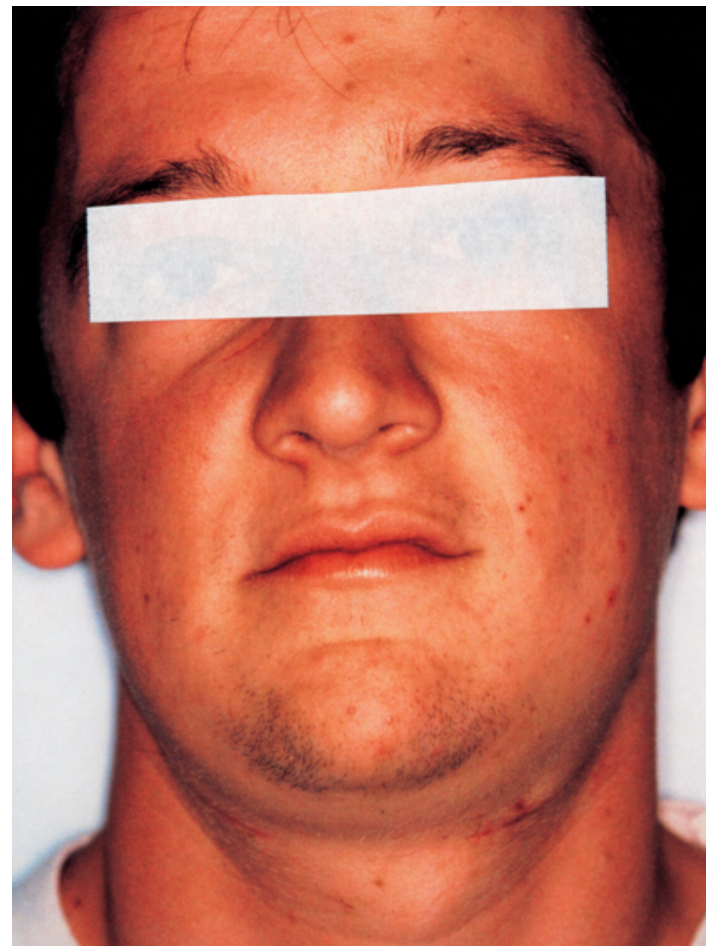

Figure 5 : Aspect clinique à une semaine post-opératoire.

Exobuccal aspect one week after surgery.

Figure 6 : Aspect clinique à un an post-opératoire. Clinical aspect one year after surgery.

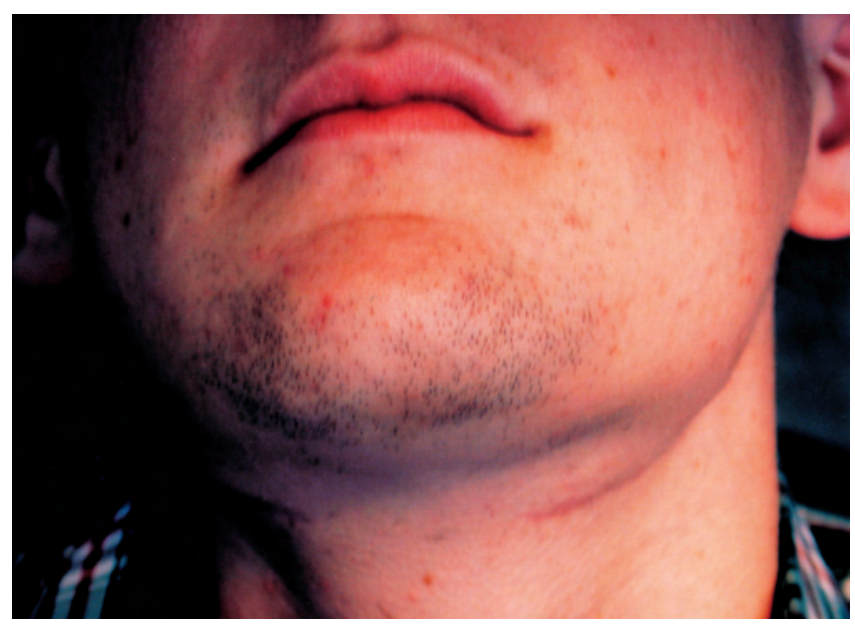

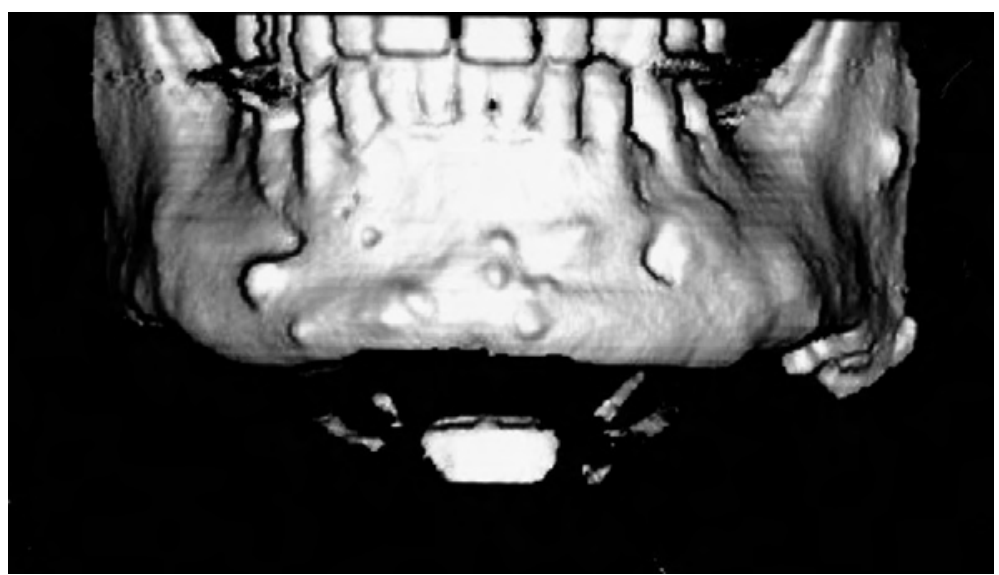

Figure 7 : Examen tomodensitométrique à un an post-opératoire montrant l'absence de récidive des ostéomes réséqués et de croissance des petits ostéomes résiduels.

An one year postoperative CT-scan revealing the absence of osteomas recurrence. 
de Gardner est situé entre 2,9 et 5,9 [16] alors que chez les sujets contrôles, le nombre d'ostéome ne dépasse pas 3. Le cas rapporté présente quatre ostéomes de grande taille et de multiples petits ostéomes. Dans le syndrome de Gardner, la surface moyenne de l'ensemble des ostéomes par patient est de $1,1 \mathrm{~cm}^{2}$ et celle de l'ostéome principal est de $\mathbf{0 , 4} \mathbf{~ c m}^{2}$ [16]. L'ostéome le plus volumineux dans le cas rapporté atteint une taille de $2 \mathrm{~cm}^{2}$. Ces ostéomes seraient présents chez $80 \%$ à $90 \%$ des patients présentant un syndrome de Gardner, alors que la prévalence des ostéomes dans la population générale est inférieure à $10 \%$ [17]. Leur radiodensité et leur ultrastructure sont celles de l'os compact mature avec un système haversien bien développé. Certains ostéomes peuvent avoir un retentissement fonctionnel tardif: compression oculaire ou nerveuse, obstruction des fosses nasales ou diminution de la mobilité. Le plus souvent, ils n'ont qu'un retentissement esthétique mais il amène le patient à consulter. Ils peuvent se développer tôt et précéder la découverte de la polypose [12]. La résection modelante est le procédé le plus fiable. Dans le syndrome de Gardner, on peut observer de multiples dents surnuméraires incluses, des odontomes complexes, des agénésies [11,16]. Jarvinen et al [19] ont trouvé des anomalies dentaires chez $18 \%$ des patients avec un syndrome de Gardner alors que seulement $1 \%$ de la population normale présente des anomalies dentaires. Dans le cas rapporté, il n'y a pas de dents surnuméraires ou de malpositions dentaires secondaires au développement des lésions osseuses. Inversement, d'autres cas de syndrome de Gardner ont été rapportés avec des anomalies dentaires mais sans d'ostéomes [20]. Ainsi, en fonction de la délétion génomique, le phénotype des atteintes au niveau de la cavité buccale peut toucher: soit le tissu dentaire (anomalies dentaires) soit le tissu osseux (ostéomes) soit les deux soit aucun des deux.

Ce travail montre l'intérêt de la chirurgie maxillofaciale dans la prise en charge fonctionnelle et esthétique des patients atteints d'un syndrome de Gardner. Les patients présentant des ostéomes volumineux souhaitent une exérèse chirurgicale. La technique d'exérèse de ces ostéomes dépend de la localisation, de la taille et de leur nombre: elle doit donc être adaptée pour chaque lésion et pour chaque patient. Le résultat esthétique et/ou fonctionnel est variable d'un cas à l'autre. Pour un patient présentant un handicap fonctionnel majeur, le résultat esthétique est secondaire. Dans le cas présenté, la voie endobuccale a permis d'obtenir un meilleur résultat esthétique mais les ostéomes du bord basilaire ont dû être traités par un abord cutané, car l'exérèse par voie endobuccale aurait été insuffisante. Les cicatrices se trouvant situées dans les plis du cou, le patient est satisfait du résultat esthétique un an après l'intervention. Seul le cas clinique présenté par Lew et al. [21], avec ostéome volumineux limitant l'ouverture buccale, a nécessité une nouvelle intervention chirurgicale à deux ans pour de nouvelles lésions osseuses faciales et crâniennes. II faut noter que notre patient à deux ans ne présente aucun nouvel ostéome et que les ostéomes non réséqués n'ont pas augmenté de taille.

La présentation d'un cas de syndrome de Gardner a permis de rappeler les différentes manifestations et expressions de ce syndrome. Selon le phénotype lié à la mutation du gène $A P C$, cette maladie est diagnostiquée plus ou moins précocement; l'âge moyen de diagnostic est d'environ 25 ans. Pour le cas rapporté, le diagnostic a été posé précocement en raison des antécédents familiaux. Ce patient a été adressé par le service de gastro-entérologie pour traiter le préjudice esthétique lié au développement d'ostéomes.

Certains signes bucco-dentaires peuvent permettre le diagnostic de cette affection. La découverte chez un sujet jeune de lésions radio-opaques des maxillaires faisant suspecter des ostéomes ou de dents surnuméraires doit faire demander une consultation de gastro-entérologie pour confirmer ou infirmer le diagnostic de syndrome de Gardner; les ostéomes précédent généralement l'apparition de la polypose intestinale. L'intérêt du diagnostic précoce est de permettre un suivi régulier des sujets atteints car l'incidence des cancers colorectaux est très élevée chez ces patients. médecine

buccale chirurgie buccale

VOL. $9, \mathrm{~N}^{\circ} 3$ 2003

page 183 


\section{RÉFÉRENCES}

1 - Devic A, Bussy MM. Un cas de polypose adénomateuse généralisée à tout l'intestin. Arch Mal App Dig 1912; 6: 278-89.

2 - GARDNER EJ. A genetic and clinical study of intestinal polyposis; a predisposing factor for carcinoma of the colon and rectum. Am J Human Genet 1951; 3: 167-76.

3 - BuLow S. Familial polyposis coli. Dan Med Bull 1987; 34: 1-15.

4 - Camuzard JF, Vaille G, Santini J, Raspaldo H, Demard F. Le syndrome de Gardner. Revue de la littérature. A propos d'une forme familiale. Ann Oto-Laryng 1990; 107: 509-13.

5 - JONES K, KORZACK P. The diagnostic significance and management of Gardner's syndrome. Br J Oral Maxillofac Surg 1990; 28: 80-4.

6 - Klemmer S, PAscoe L, De Cosse J. Occurrence of desmoids in patients with familial adenomatous polyposis of the colon. Am J Med Genet 1987; 28: 385-92.

7 - SMITH WG. Familial multiple polyposis: research tool for investigating the etiology of carcinoma of the colon?

médecine buccale chirurgie buccale

VOL. $9, \mathrm{~N}^{\circ} 3$ 2003

page 184 Dis Colon Rectum 1968; 11: 17-31.

8 - Herve R, Farret O, Mayaudon H, Helie C, Denee JM, Bauduceau B, Molinie C. Association syndrome de Gardner et carcinome thyroïdien. Presse Med. 1995; 24: 415

9 - BLAIR NP, TREMPE CL. Hypertrophy of the retinal pigment epithelium associated with Gardner's syndrome. Am J Ophthalmol 1980; 90: 661-7.

10 - Traboulsi El, Krush AJ, Gardner EJ, BoOker SV, Offerhaus GJ, Yardley JH, Hamilton SR, LuK GD, GiARDIELlo FM, WelSH SB. Prevalence and importance of pigmented ocular fundus lesions in Gardner's syndrome. N Engl J Med 1987; 316: 661-7.

11 - CARL W, HeRrera L. Dental and bone abnormalities in patients with familial polyposis coli. Semin Surg Oncol 1987; 3: 77-83.
12 - Chang CH, Piatt ED, Thomas KE, Watne Al. Bone abnormalities in Gardner's syndrome. Am J Roentgenol Radium Ther Nucl Med 1968; 103: 645-52.

13 - Karazivan M, Manoukian K, LaLONDE B. La polypose adénomateuse familiale ou syndrome de Gardner. Revue de la littérature et présentation de deux cas cliniques. J Can Dent Assoc 2000; 66: 26-30.

14 - Ruhin B, Gentile PF, Rose C, Leclerce A, Ferri J. Syndrome de Gardner. Prise en charge maxillo-faciale d'une forme évolutive extrême. Rev Stomatol Chir Maxillofac 1998; 99: 235-40.

15 - TAKEUCHI T, TAKENOSHITA Y, KUBO K, IIDA M. Natural course of jaw lesions in patients with familial adenomatosis coli (Gardner's syndrome). Int J Oral Maxillofac Surg 1993; 22: 226-30.

16 - Katou F, Motegi K, Baba S. Mandibular lesions in patients with adenomatosis coli. J Craniomaxillofac Surg 1989; 17: 354-8.

17 - UTSUNOMIYA J, NAKAMURA T. The occult osteomatous changes in the mandible in patients with familial polyposis coli. Br J Surg 1975; 62: 45-51.

18 - FAdER M, KLINE SN, SpATZ SS, ZUBROW HJ. Gardner's syndrome (intestinal polyposis, osteomas, sebaceous cysts) and a new dental discovery. Oral Surg Oral Med Oral Pathol 1962; 15: 153-72.

19 - Jarvinen HJ, Peltokallio P, Landtman M, Wolf J. Gardner's stigmas in patients with familial adenomatosis coli. Br J Surg 1982; 69: 718-21.

20 - Nahas Gholmieh M, Nasseh I, Sayegh R, Sfeir E. Le syndrome de Gardner : une expression orale familiale particulière. Actual Odonto Stomatol 1997; 199: 593-8.

21 - Lew D, DewitT A, Hicks RJ, Cavalcanti MG. Osteomas of the condyle associated with Gardner's syndrome causing limited mandibular movement. J Oral Maxillofac Surg 1999; 57: 1004-9. 